EPJ Web of Conferences 92,02095 (2015)

DOI: $10.1051 /$ epjconf/ 20159202095

(c) Owned by the authors, published by EDP Sciences, 2015

\title{
Colliding wall-jets on a cylindrical surface
}

\author{
Václav Tesar̆ ${ }^{1, a}$, Kazimierz Peszynski ${ }^{2}$ \\ ${ }^{1}$ Institute of Thermomechanics v.v.i., Academy of Sciences of the Czech Republic, Prague, Czech Republic \\ ${ }^{2}$ University of Technology and Life Sciences, Bydgoszcz, Poland
}

\begin{abstract}
Paper discusses aerodynamics and potential engineering applications of an unusual and in literature practically unknown fluid flow configuration, with two wall-jets attached to a cylindrical surface so that they collide head-on and by mutual conjunction generate a single jet directed away from the wall. Applications are envisaged in pneumatic sensors, particularly those operating at low Reynolds numbers. Performed experimental investigation, combined with numerical flowfield computations, revealed several interesting aspects. The most interesting among them is the discovery of symmetry-breaking existence of three different stable flow regimes. This opens a possibility for fluidic tristable amplifiers and systems operating with ternary logic.
\end{abstract}

\section{Introduction}

The key problem of fluidics — the technique of handling fluids without action of mechanical components - is how to set up a useful flowfield that responds by a substantial change of their character to a weak control flow input. A typical example of such sensitive flow is the jet used in diverter type fluidic amplifiers. A small momentum of the control jet acting in the sensitive region near the nozzle exit can change substantially the direction into which the jet flows. Another, less often used sensitive flowfield is set up by head-on collision of two jets. Of high importance to fluidic amplifiers are wall-jets - generated by fluid flow issuing from a nozzle and on one side bounded by a wall. Wall-jets are used in the very popular fluidic diverter amplifiers based on the Coandaeffect attachment of a jet to an adjacent wall.

There are intriguing flowfield configurations having a certain relation to both colliding jets and wall jets, yet so far at the periphery of interest. One such case are colliding wall-jets. They are so sensitive to the ratio of their momenta that it may be difficult to set them up into the balanced state with the stagnation point of collision in the middle distance between two geometrically identical opposed nozzles. The solution is to supply both nozzles from the same fluid source. This is easy to arrange in the case of colliding wall-jets curved by Coanda-effect attachment to the same cylindrical surface, as presented in Fig. 1.This flow configuration is simply set up by placing a cylindrical body between the lips of a very wide nozzle exit, leaving a slit on both sides.

Present paper discusses this so far little known and little used flow configuration, which recently found useful application in fluidics. The high sensitivity made it useful for fluidic sensors of mechanical displacements. In

a Corresponding author: tesar@it.cas.cz literature is information about the colliding curved wall jets practically non-existent. There is, in fact, not much information available on the less intriguing subject of collision straight jets, in [1, 2, 3, 4]. Ref. [13] discusses the violent flow pulsation that appears due to instability. In an application discussed in [14] one of the jets turns down the outflow from the opposing nozzle.

Attachment of a wall-jet to a cylindrical surface without the other, opposing wall-jet - contains itself several problems, as discussed e.g., in [7, 8, 15]. Another point of view that may contain useful information is the case of colliding straight wall-jets in $[5,6]$. The present paper thus aims at filling the existing gap. The approach is two-pronged. On one hand there were experimental investigations using a scaled-up laboratory model. The scaling beyond the scales typical in fluidics was necessary for an access into the critical parts of the flowfield with a probe of reasonable size. Even so not all variables of interest could be measured without disturbing the flowfield. Therefore, the experimental approach was supplemented by numerical flowfield computations where such disturbances by probes are absent.

\section{Experiments}

The basic features of the model used for the measurements are presented in Fig. 1. The design of the cavities was two-dimensional, planar - which, of course, does not mean that the flowfield is two-dimensional (as will be shown below, there are significant threedimensionalities). The height of all cavities was everywhere the same, $\mathrm{h}=8 \mathrm{~mm}$. This was the distance between two plane top and bottom end plates. The latter 


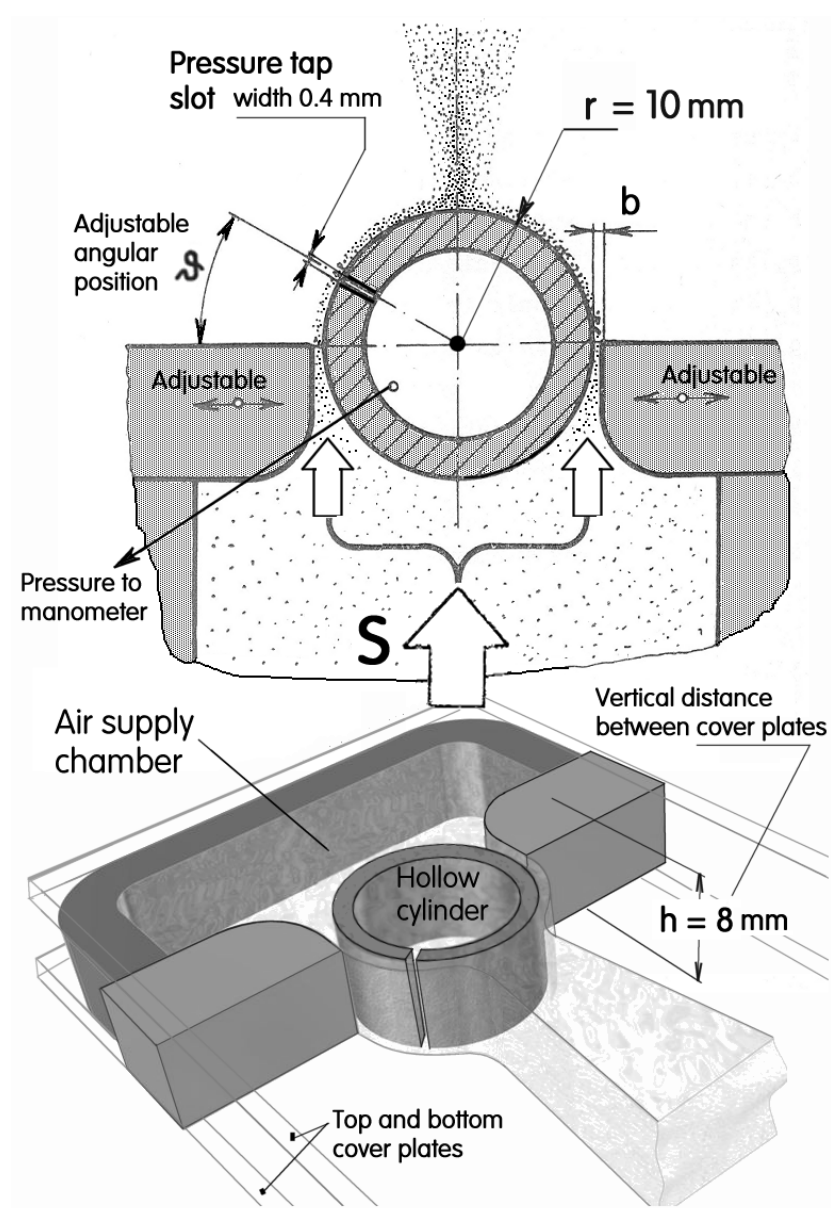

Figure 1. Two wall-jets, issuing through parallel slit-shaped nozzles from the common supply chamber, are curved by attachment to cylindrical surface of the disk-shaped central body. The curvature leads to their head-on collision. From there is generated the resultant jet, very sensitive to minute changes in the ratio of jets' momentum flow rates.

were made of Perspex to make possible flowfield observation. The essential part was the wide nozzle with its adjustable left and right rounded lips. Into the central position between these lips was inserted the disk of $d=$ $20 \mathrm{~mm}$ diameter. The width $\mathrm{b}$ of the resultant two walljet generating nozzles was adjusted by moving the nozzle lips.

Of critical influence on the strength of the Coandaeffect attachment to the cylindrical surface of the central disk is the relative curvature characterised by the ratio $b / r$ $(r=d / 2)$, Because of the limited available time for the laboratory tests, only two nozzle slit widths $b$ could be adjusted, either $b=1 \mathrm{~mm}$ or $b=0.5 \mathrm{~mm}$. This means there was either the smaller relative curvature radius $\mathrm{r} / \mathrm{b}$ $=10$ or the larger $\mathrm{r} / \mathrm{b}=20$.

Two basic objectives of the investigation were the pressure distribution measurements on the disk surface and velocity profiles of the generated resultant jet leaving the central part of the model. For the pressure measurements was made in the disk a thin slot serving as pressure tap, of width $0.4 \mathrm{~mm}$, shown in Fig. 1. The pressure from this tap entered the internal cavity of the disk and from there was led into a micromanometer.
Of course, the advantage of the circular shape of the disk body is the single tap slit suffices, by rotating the disk into different angular positions, characterised by the angle $\boldsymbol{N}$. The slit version of the pressure tap (rather than the more usual round hole) performed an integration of the pressure readings along the whole height $h$ and thus it extracted the local mean value at a particular angular position.

Mass flow rate $\dot{M}\left[\mathrm{~m}^{3} / \mathrm{s}\right]$ of the supplied air prior to entering the model was measured by a standard laboratory flowmeter. An important quantity for characterisation of the flow is the nominal nozzle exit velocity $w_{e}[\mathrm{~m} / \mathrm{s}]$. It is evaluated from the air flow rate and the dimensions $b$ (width) and $h$ (height) of the nozzle exit - and the specific volume $v\left[\mathrm{~m}^{3} / \mathrm{kg}\right]$ of the air:

$$
w_{e}=\dot{M} v /(b . h)
$$

This definition neglects the existence of boundary layer formed on the internal walls of the nozzle (and also on the top and bottom endplates).

Two geometrically identical flowfields generated by the nozzle flows are mutually similar if they have the same value of Reynolds number

$$
\operatorname{Re}=\frac{w_{e} b}{\nu}
$$

- where $\nu\left[\mathrm{m}^{2} / \mathrm{s}\right]$ is fluid viscosity and .

An example of the surface pressure readings for $\mathrm{r} / \mathrm{b}$ $=20$ at different Reynolds numbers is presented in Fig. 2. Over the majority of the range of angles $\boldsymbol{S}$ the pressure difference $\Delta \mathrm{P}_{\mathrm{w}}[\mathrm{Pa}]$ between the surface and the atmosphere is negative - the corresponding pressure force oriented towards the lower pressure at the attachment surface holds, as the Coanda effect, the air jet attached. Only in the vicinity of the central angular position $\boldsymbol{A}=\pi / 2$ (at the same adjustment of both nozzles and therefore same momentum flow rates) the sign of the pressure became positive. This sign change is the consequence of the pressure rise to the stagnation pressure value in the collision location.

Apparent in Fig. 2 is the inconvenience of graphical presentation widely different absolute values of the measured pressure differences at different Reynolds numbers. It makes difficult an inspection that would reveal the Reynolds number effects. A much better presentation is obtained by non-dimensionalisation plotting the much less mutually differing values of the pressure coefficient

$$
c_{P}=\frac{2 v \Delta P_{w}}{w_{e}^{2}}
$$

Measurements of flows in the two different geometries of the active cavities - with the relative curvature radius either $r / b=10$ or $r / b=20-$ show that the resultant two data sets are also mutually incompatible. Their correspondence becomes much neater by using the modified pressure coefficient $\mathrm{C}_{\mathrm{P}} *$ defined

$$
c_{\mathrm{P}}^{*}=\frac{2 \mathrm{v} \Delta \mathrm{P} r}{\mathrm{w}_{\mathrm{e}}{ }^{2} \mathrm{~b}}
$$

An example of wall pressure measurement presented in terms of this variable is shown in Fig. 3. The 


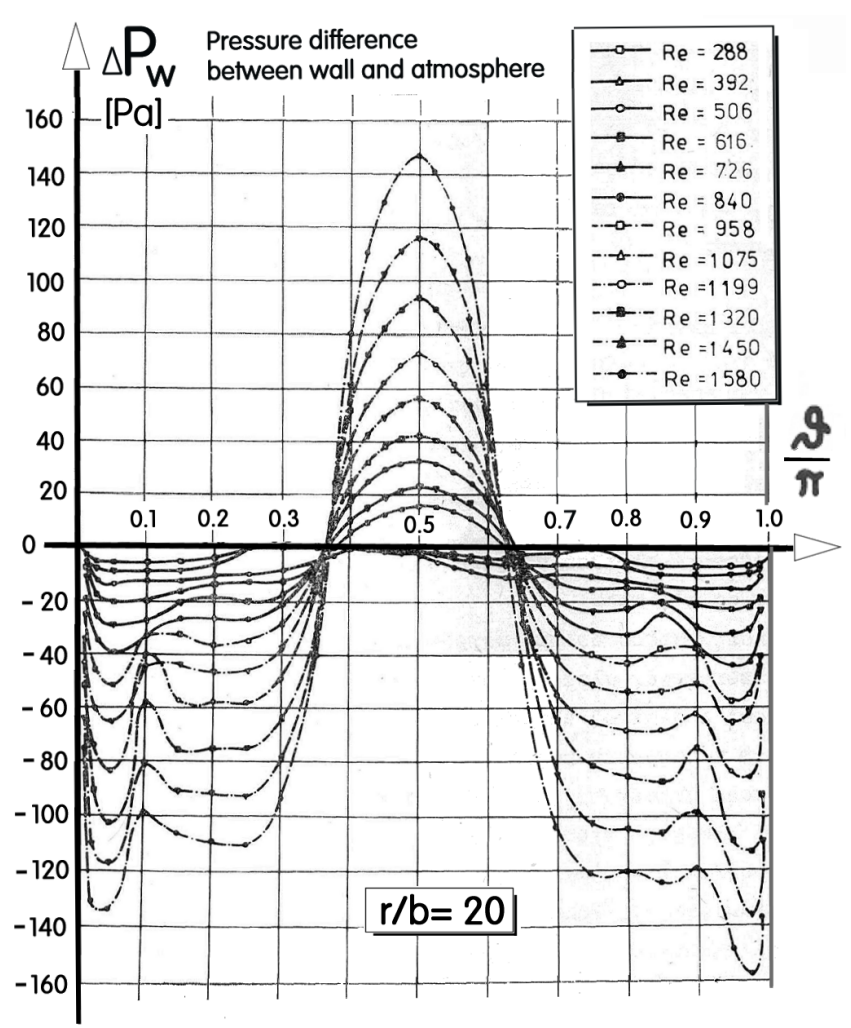

Figure 2. Measured pressure distributions on the surface of the central cylinder at different Reynolds numbers. Width $b$ of both nozzles was adjusted to $0.5 \mathrm{~mm}$.

use of this non-dimensional variable for investigating the effect of Reynolds numbers is shown in the next Fig. 4. If there were a perfect Reynolds number invariance, the three data sets obtained at different air flow rates would collapse into a single universal curve. The fundamental task of Fluid Mechanics science is search for invariants in the investigated processes. Unfortunately, Fig. 4 demonstrates that in the investigated problem this invariance is not obtained. On the other hand, it is evident that the difference between the three plotted curves is not

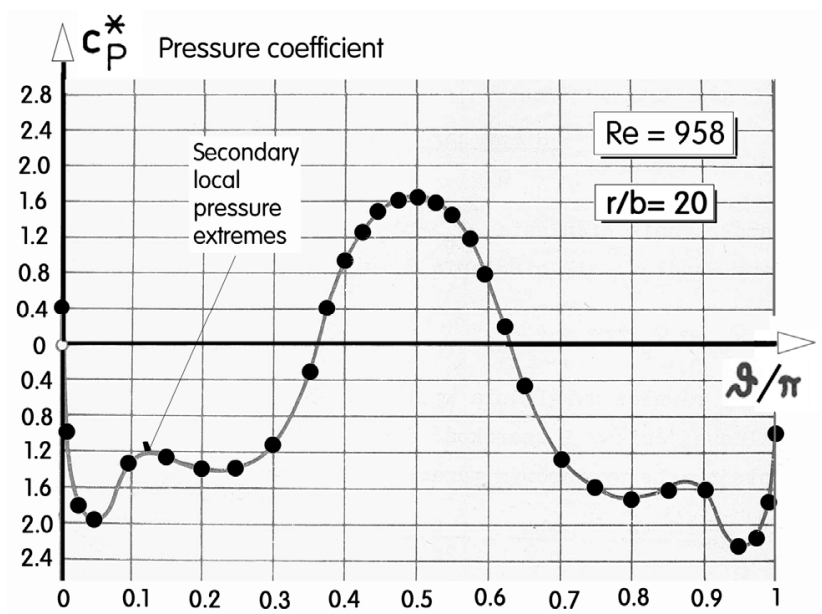

Figure 3. To suppress the wide differences in values (so that they may be conveniently plotted in the same diagram), the measured pressure was converted into dimensionless pressure coefficient $\mathrm{C}_{\mathrm{P}} *$ eq.(4) here evaluated for one of the distributions in Figure 2.

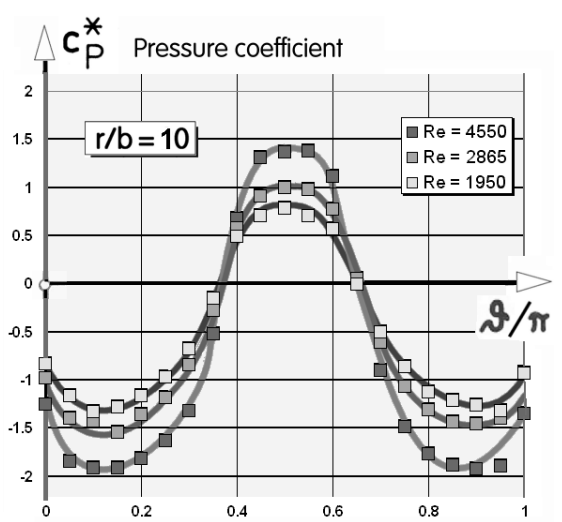

Figure 4. Demonstration example of the pressure coefficient making the plotted values at different flow rates mutually comparable - but not equal.

very large. It might be perhaps even neglected in a first approximation to the problem by plotting a mean line passing through all data points, considering the deviations to be just data scatter. Although not strictly correct, this neglect of not excessive Re-dependent deviations and evaluation of the mean curve may suffice for engineering design purposes, such as e.g. estimation of the wall pressure - at least in the given range of Fig. 4 from $\mathrm{Re}$ $\sim 1.910^{3}$ to $\operatorname{Re} \sim 5.010^{3}$.

In addition to the central cylinder surface pressure measurement also investigated were the generated flowfields, especially the velocity profiles of the resultant jet. They were measured by traversing Pitot-type probe. For the intended purpose of understanding the time-mean conditions in the flow the Pitot probe data were sufficient. The geometry of the probe tip as well as the arrangement of the traversing probe holder are presented in the next Fig. 5.

\section{Knob for fixing the angular position}

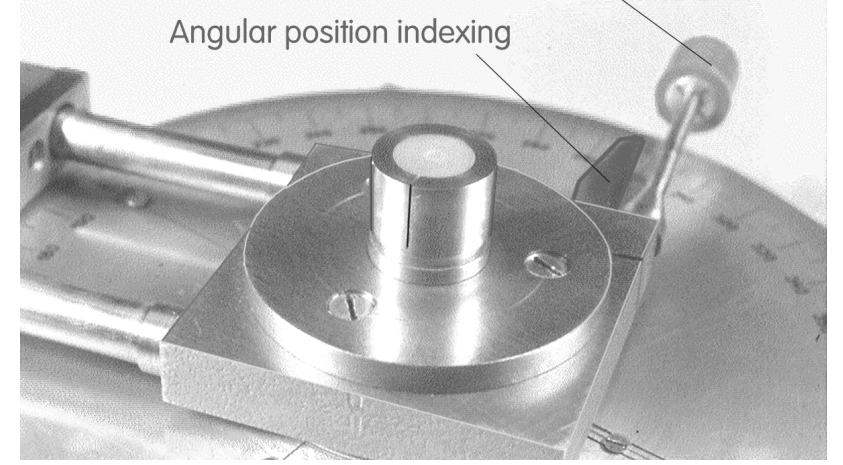

Figure 5. Photograph of the central cylinder and the angular position adjustment components. The parts with nozzles and air supply chamber are here removed.

\section{Numerical flowfield computations}

Since not all aspects of the flowfield inside the model were accessible for direct probing, it was decided to complement the measurements by numerical computations of the flowfield. This has the advantage of unlimited accessibility to any point (or, for that matter, a 


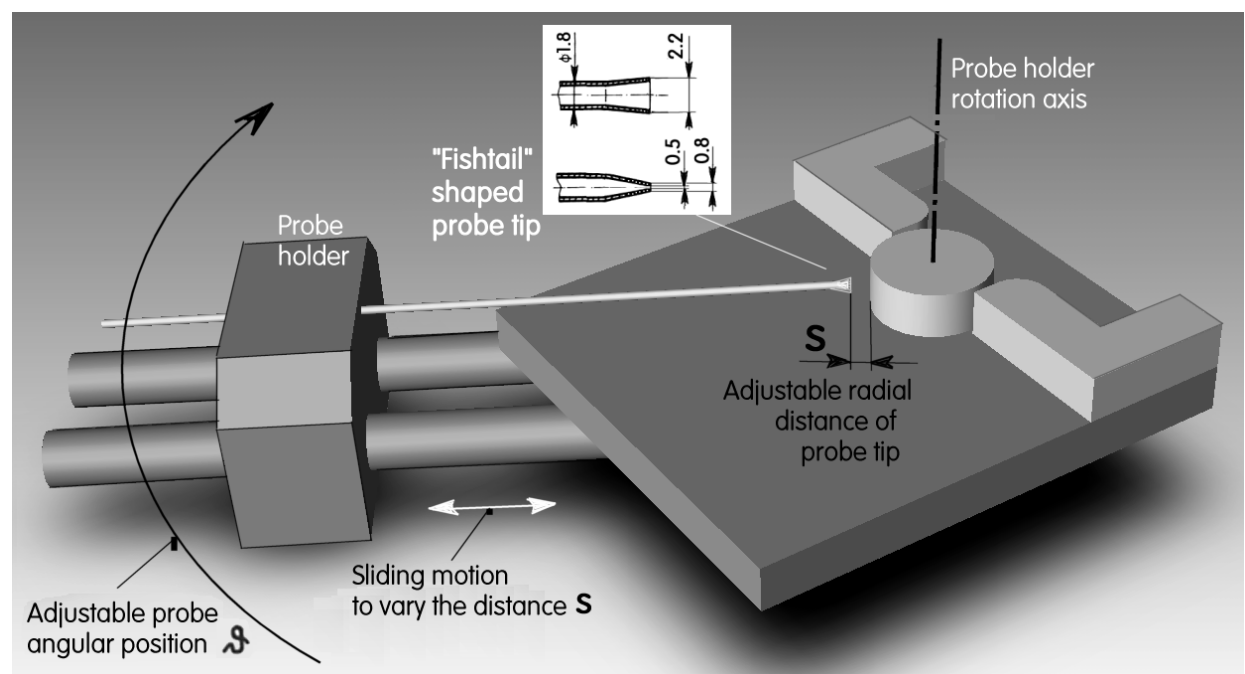

Figure 6. For investigating the flowfield - and mainly the generated resultant jet - was used Pitot probe of the "fishtail" shape (detail in the inset on the top of the picture) fixed to rotated and radially shifted holder. In most measurements the adjusted radial distance $\mathbf{S}$ from the surface of the central disk was kept constant and the probe was systematically moved at small increments of the angles $\$$ defined by zero value (Fig. 1) at the nozzle lip.

line or a surface) inside the computation domain. It is also much more easy and fast to make changes of model geometry in the abstract mathematical model than in a laboratory model that requires metal cutting in the workshop. On the other hand, it has to be expected that the numerical solutions are sometimes less reliable particularly in those cases, like the present one, characterised by rather low Reynolds numbers of the order (here from $10^{2}$ to $10^{3}$ ) where the success depends very much upon proper modelling the inception and early development stages of turbulence. These phenomena cannot be so far fully reliably modelled by mere adaptation of existing developed-turbulence models. Experience has thus shown as the best approach to combine the experiment and computations - with adjustment of the computational procedures so that they fit reasonably to a selected aspect of the measurements.

This combined approach was also used in the present case. Two sets of laboratory data were chosen for the matching. The first one is the pressure distribution presented in Fig. 3. The other case was the profile of radial velocity as will be presented below in Fig. 9. The computations used standard FLUENT finite volumes software in computation domain discretised by unstructured tetrahedral-elements grid set up by means of software package GAMBIT. The computational model was fully three-dimensional - despite the apparent twodimensionality of the model geometry, with the cavities height $h$ constant everywhere. Especially the effect of the boundary layers on the top and bottom end-plate surfaces were strongly three-dimensional. Also, in spite of the mirror symmetry of the flowfield, the solution was undertaken without this symmetry taken into account (a factor that later made possible also computations with the jet deflection.

The solution domain corresponded to that of the laboratory model as it is shown in Fig. 1, i.e. with the diameter of the cylindrical disk $d=20 \mathrm{~mm}$ and height $\mathrm{h}=8 \mathrm{~mm}$ constant everywhere. The space between the endplates downstream from the nozzle exit, in which was computed the jet resultant from the wall-jets collision and merging, was $60 \mathrm{~mm}$ long and $80 \mathrm{~mm}$ wide. On the two $60 \mathrm{~mm} \times 8 \mathrm{~mm}$ boundaries of the domain as well as on the single $80 \mathrm{~mm} \times 8 \mathrm{~mm}$ plane were defined boundary conditions of constant atmospheric pressure. At the inlet, into the air supply chamber (Fig. 1) was defined velocity boundary condition. The total volume of the computation domain was $4.5110^{-5} \mathrm{~m}^{3}$. Its discretisation was uneven. In the region inside the wall-jet nozzles and their vicinity, as well as at the cylindrical surface of the disk, the finite volumes were very small and gradually decreased in the course of the computation steps. Elsewhere the initial division into the finite volumes was more coarse. There were initially $242000 \mathrm{f}$ tetrahedral cells. The refinement (using its unstructured character of the grid) was by decreasing the cell size in those regions where the gradients of absolute velocity were larger than $0.012 \mathrm{1} / \mathrm{s}$. As a result, typical final number of the elements was mostly near to 260000 (different in different computation runs). In this final domain condition, the minimum volume of the mesh elements was $8.5 \times 10^{-13}$ $\mathrm{m}^{3}$ (i.e. $1.9 \times 10^{-8}$ of the total domain volume) while the maximum volume of elements was $3.82 \times 10^{-8} \mathrm{~m}^{3}$ (equal to $8.43 \times 10^{-4}$ of the total). Minimum face area of a cell was $1.9 \times 10^{-8} \mathrm{~m}^{2}$ (inside the nozzles), while the maximum area was $2.95 \times 10^{-5}$. The computations were run as long as the gradually decreasing relative value of the equations residua (i.e. inequalities between the lefthand and right-hand sides of the solved equations for momentum and turbulence transport) decreased to the limit set at $10^{-4}$. The computation was performed using the two-equation $k-\varepsilon$ model of turbulence, modified in the locations of low Reynolds number of turbulence by the RNG procedure, provided by the software supplier. 


\section{Correlation of experiment and computation}

As mentioned above, there were two test cases for correlating the computation with results of experiments. The first one was the adjustment of pressure computations so that the corresponding numerical solution were fitted to the experimental data presented in

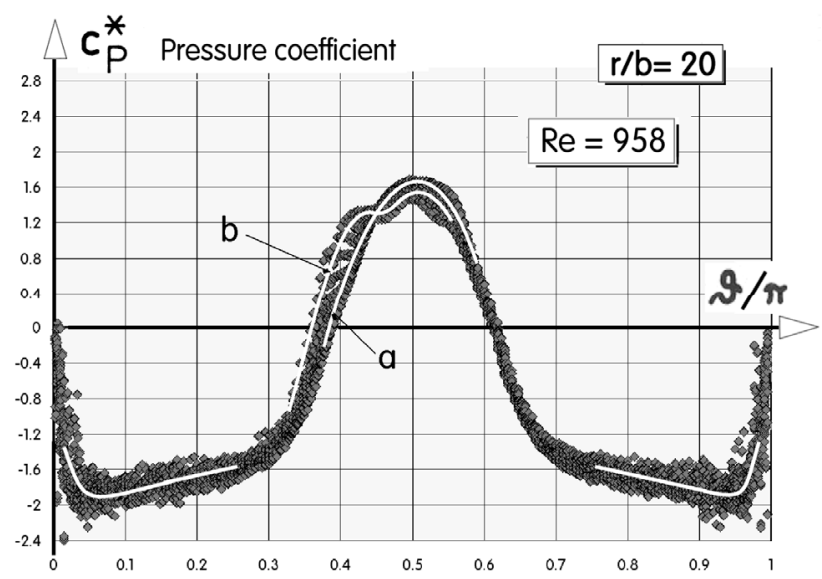

Figure 7. Pressure distributions on the surface of the central disk - computations for the same conditions as in the experiment the result of which are presented in Figure 3.

Fig. 7, i.e. at low Reynolds number slightly less than $\mathrm{Re}$ $=1000$, obtained with air flows from the two $b=0.5$ $\mathrm{mm}$ wide nozzles. The other comparison and adjustment case was the correlation between the measured and computed velocity profiles presented in Fig. 9, for Reynolds number $\mathrm{Re}=2$ 254. Of course, the adjustments in these two cases did not ensure a complete agreement in other cases, but - apart from one significant deviation to be discussed - the agreement of computed and measured values could be characterised as quite good. The fitting actually required only minimal, insignificant adjustments of turbulence model constants.

The computed data values corresponding to the experimental data in Fig. 3 are presented in Fig. 7. The total number of the computed data points plotted in the diagram was 12 810. (this is why they seem to cover in Fig. 7 a continuous area). While the slit arrangement of the pressure tap generated a single mean value at each angular position, the computations evaluated different local pressure values at different data point positions along the height $h$. This was an addition to the usual scatter. Inspection of Figs. 3 and 7 shows that the mutual agreement between them is quite good.

1) The white lines $a$ and $b$ drawn on the background of data points in Fig. 7 show the mean values for the computed data. Somewhat surprisingly, there is not a single mean line. Instead, in the middle of the diagram there are two. One of them, a, is symmetric while the other, $b$, is slightly asymmetric. The search for explanations has shown that the flows were actually never fully symmetric (despite the symmetry of the domain geometry) and there was in details of the compu-

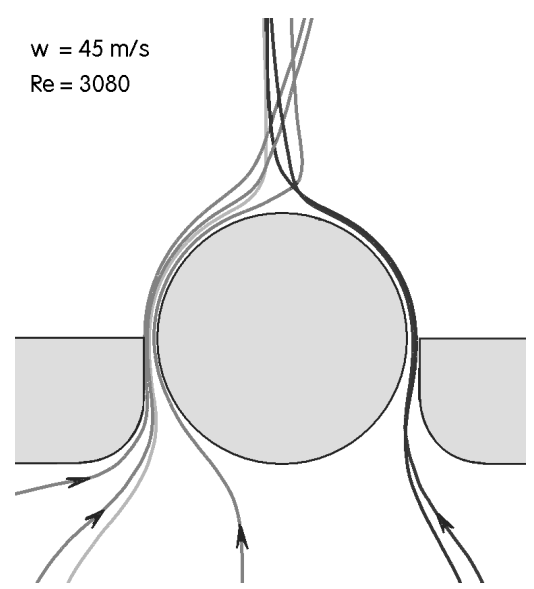

Figure 8. Computed pathlines downstream from the central disk reveal the complex three-dimensionality due to the faster one of the two wall jets entering the end-plate boundary layers. This is apparently the reason behind the difficulties encountered when adjusting the symmetric flow in experiments.

ted flowfield, as presented in Fig. 8, a small but significant three-dimensionality. Contrary to the expected symmetric collision of the two flows, with simply straight vertical line of stagnation points, the computed pathlines show that the one of the wall-jets could be slightly faster and managed to find its way further away along the angular distance over the disk surface, beyond the nominal stagnation location. By inclination (not seen in the two-dimensional projection presented in Fig. 8) this flow found slower opposing flow in the boundary layers (formed on the top and bottom cover plates). The white lines $a$ and $b$ obviously show the consequence of the resultant partial asymmetry.

2) The other difference, initially considered (mistakenly) to be less important, is the absence in the computational results of the secondary extremes indicated in Fig. 3. These extremes were found to be a consequence of the laminar/turbulent transition, which here takes place at lower Reynolds numbers than is the case in flows

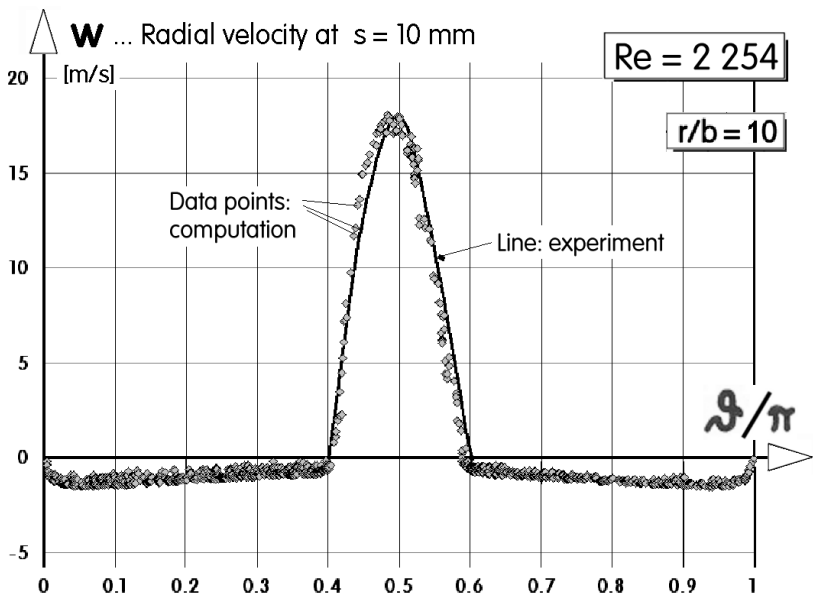

Figure 9. Comparison of measured and computed velocity component perpendicular to the cylindrical surface $\mathrm{s}=10 \mathrm{~mm}=$ const. Equally as in the comparison of Figs. 3 and 7 there is a general mutual agreement between the computed and measured distributions - with one important exception discussed below. 
without significant curvature of pathlines. At the Reynolds number $\mathrm{Re}=958$ for which were computed and measured the data in Figs. 3 and 7, the flowfield with straight flowpaths would be laminar - or at most containing organised eddies (which the two-equation averaged model cannot distinguish from turbulence). Such low Re jet flows are unlikely to attach to the curved surface because of weak entrainment of outer fluid. It is the entrainment that causes the pressure difference on which the jet deflection and Coanda effect depends. Such insufficient attachment would manifest itself in the pressure distributions on the wall by local increase of the pressure coefficient $\mathrm{C}_{\mathrm{P}}{ }^{*}$ values. Such trend to the increase is seen in Fig. 3. Before it can rise to positive $C_{\mathrm{p}}{ }^{*}$ values indicative of separation, the early transition into turbulence limits it to only a small local pressure extreme. The used FLUENT variant, while computing the pressure distribution reasonably well, fails to show properly the effect of early laminar/turbulent transition. The local extremes from Fig. 3 are absent in Fig. 7 at both righthand and left-hand extremes of the diagram.

Concerning the velocity profiles, the comparison between the velocity measured by the Pitot probe and the computation results is presented in the example case in Fig. 9. Plotted there as a function of the angular position are the magnitudes of radial velocity at the distance $\mathrm{s}=$ $10 \mathrm{~mm}$ from the cylindrical surface. Of course, Pitot probe could measure only the positive radial velocities, i.e. those of the air flow directed away from the disk. The measured values are therefore seen only in the centre of the diagram, where the comparison with computations documents a good mutual correlation - over the rather small range, $0.2 \pi$ radians in total width, at the centre of the diagram. Elsewhere there is a suction from the atmosphere towards the attachment surface, represented by negative velocity - which the probe was unable to detect. While the height and overall shape of the positive radial velocity profiles may be described as almost excellent, the computations exhibit a slight asymmetry (shift of the profile to the left), probably due to the phenomena related to those indicated in Fig. 8.

\section{Jet deflection in response to shifting the central disk}

With the reliability of the computations established, it was possible to investigate various aspects of the flowfield of colliding wall-jets by numerical solutions. In view of possible applications in pneumatic sensors, one of the computation series was aimed at determining the sensitivity of the flowfield to small imbalance made by moving the disk in small steps transversally. Figure 10 shows one example of the computed response. Shifting the disk of diameter $\mathrm{d}=20 \mathrm{~mm}$ by the distance of only $\mathrm{x}$ $=0.4 \mathrm{~mm}$ (i.e. by mere $\mathrm{x} / \mathrm{d}=0.02$ ) the generated jet is seen to be deflected to the very large angle $37 \mathrm{deg}$. This is a very large value. Already a deflection smaller by a decimal order of magnitude (i.e. 2 -3 deg) would suffice for generating a fluidic signal suitable for processing in an attached fluidic circuit.

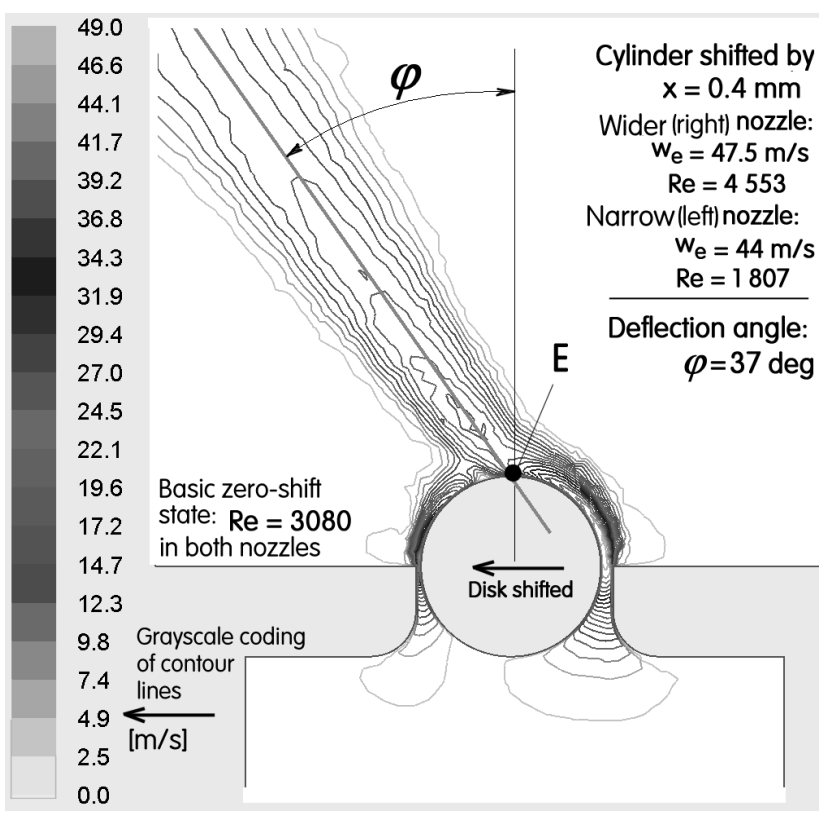

Figure 10. An example of computed asymmetric flowfield with asymmetry caused by shifted central disk. This large angular response of the resultant jet generated by the merger of the two colliding wall-jets to the shift as an input is used in fluidic sensor of mechanical motion [11].

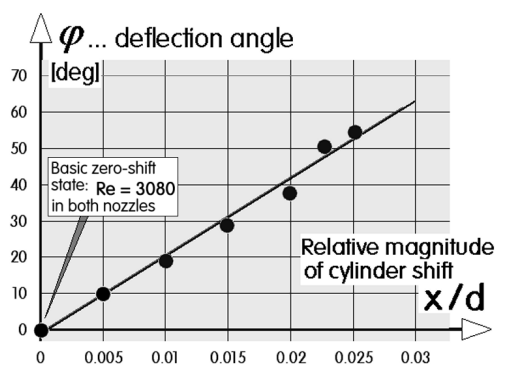

Figure 11. Strong dependence of the resultant jet inclination on displacement $\mathrm{x}$ of the disk (Figure 10).

The results of seven computed cases with various magnitudes $\mathrm{X}$ of the shift are presented in Fig. 11. For unsteady sensing applications it may be an important fact that the dependence in this diagram is linear. The angles $\varphi_{\text {were evaluated (as in Fig. 10) from straight lines fitted }}$ to the computed contours of local absolute velocity magnitude.

An interesting fact apparent in Fig. 10 is the location of the origin of this fitted straight line. It does not pass through the centre of the disk, which may be the naturally expected origin. Instead, it passes through the point $E$ at the top of the disk.

A presentation analogous to that in Fig. 10 was also chosen in Fig. 12 to show computed distribution of specific kinetic energy $e_{f}$ of unsteady motions inside the jet (i.e. the combined effect of turbulence and coherent vortices - the two indistinguishable in the used mathematical model). The presentation in Fig. 12 was computed for the same Reynolds number value as was the velocity field in Fig. 10. Both were evaluated in the midplane, i.e. the plane parallel with the top and bottom end plates, positioned at $4 \mathrm{~mm}$ above the bottom end plate (cf. Fig. 5). An interesting fact visible in Fig. 12 is 


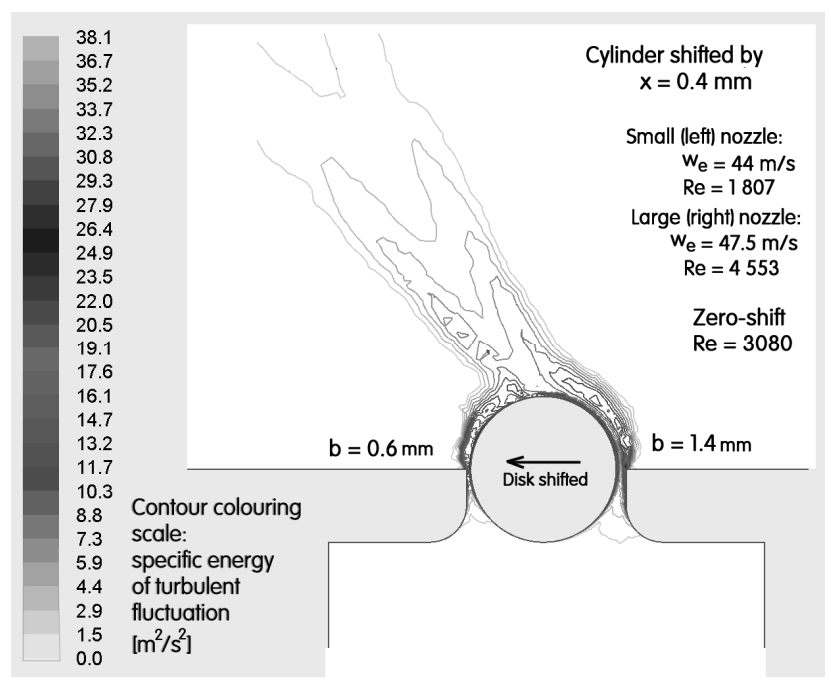

Figure 12. Computed distribution of the specific energy of fluctuation in the flowfield from Figure 10. Note the trough of local minima on the axis of the deflected jet. Considering the relatively low Reynolds number value the intensity of fluctuation is surprisingly large.

the local maxima of kinetic energy of the fluctuation in the resultant jet. These maxima have two "tongues", with a distinct local minimum between them. The Reynolds numbers of the flow in both nozzles (they are in this particular case different, the values are indicated in Fig. 12) are quite low. Nevertheless, these values are not as low as to eliminate a possibility of turbulent character of the flowfield even with jet flows not affected by the promotion of the transition. It was therefore considered instructive to present also another computation results of the distribution of the fluctuation energy — for the

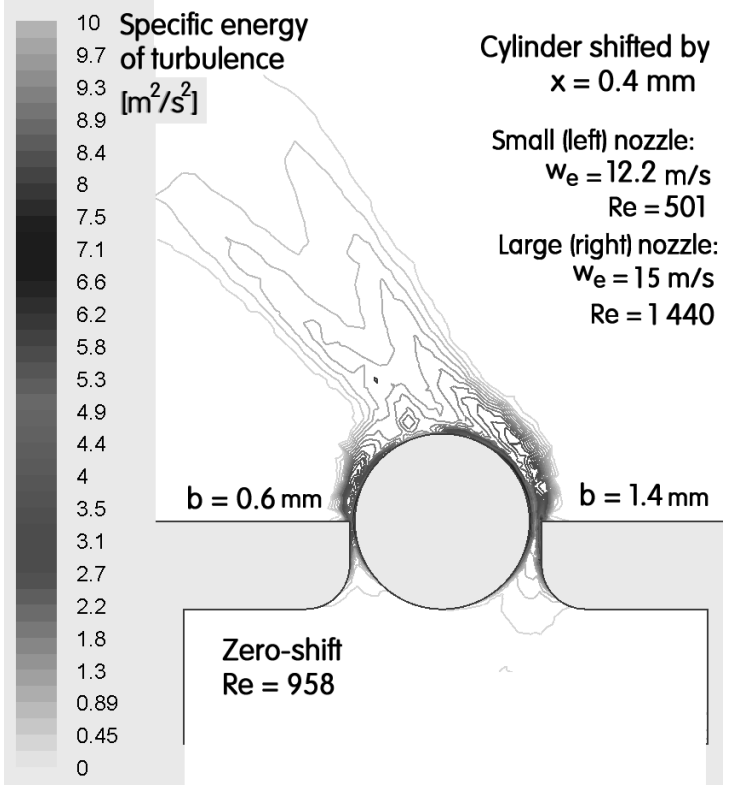

Figure 13. Distribution of the turbulence energy under conditions differing from the case presented in Figure 12 by lower Reynolds number - so low that a straight-pathline flow at this $\operatorname{Re}$ would be laminar. The pathline curvature causes an earlier laminar-to-turbulent transition. conditions of even substantially lower Reynolds number, conditions at which the two nozzle flows (note the Reynolds number value only $\operatorname{Re}=501$ for the smaller one of the two nozzles). At these conditions it might be reasonably expected (on the basis of knowledge about straight flows) that the flowfield will be purely laminar. Nevertheless, turbulence is seen not only to be present but to be quite fierce. This is another demonstration of the above already discussed fact that curvature of flow pathlines causes an earlier transition into turbulence.

The conclusion that the turbulence is promoted by the flow curvature is apparent also from the incongruence in the two cases the specific kinetic energy of fluctuation $e_{f}$ with the specific kinetic energy $e_{k}=w_{e}^{2} / 2$ of the time-mean flow inside the narrower one of the two nozzles. In the higher Reynolds number case of Fig 11 (in the right-hand side nozzle where there is $R e=1807$ ) the ratio $e_{f} / e_{k}$ is 0.039 . In the case of Fig. 12 with the low Reynolds number only $\operatorname{Re}=501$, at which might be expected laminar flow, the relative fluctuation energy $e_{f} / e_{k}$ is much higher 0.134 . Obviously, this high relative fluctuation level is not caused by the natural hydrodynamic instability of the basic flow but some other turbulence-promoting factor, of which all indications point to the influence of secondary streamwise vortices in the outer layer of the wall-jets.

\section{Surprising three stable regimes \\ 6. 1 Discovered phenomenon of symmetry breakdown}

The most interesting finding in the discussed project was the discovery of a breakdown of symmetry. While the geometry of the laboratory model was completely symmetric - and also symmetric were the boundary conditions of velocities and pressures - the resultant jet was sometimes found to remain inclined with no obvious reason. The deflection was stable when exposed to disturbances of small to medium scale The deflection angle $\varphi$, was quite large, around $0.25 \pi$. The resistance to disturbances was practically equal to what was earlier

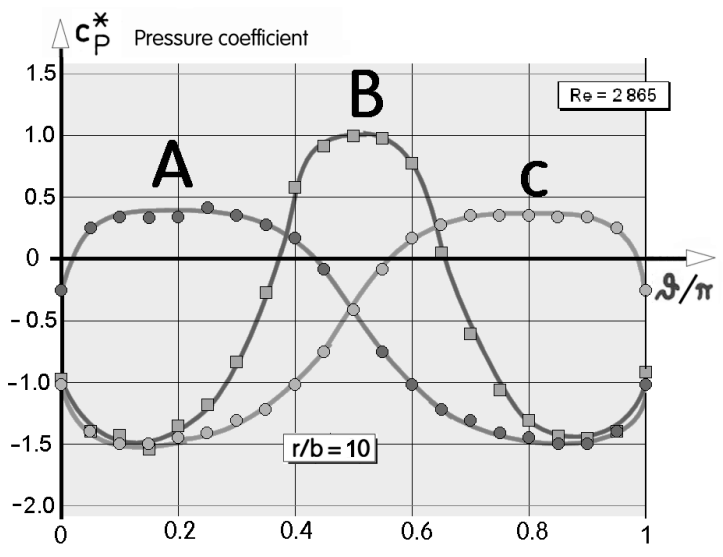

Figure 14. The surprising fact discovered in experiments with the configuration having smaller relative curvature radius $\mathrm{r} / \mathrm{b}$ : existence of three different stable flow regimes, here labelled $A$, $\mathrm{B}$, and $\mathrm{C}$. 


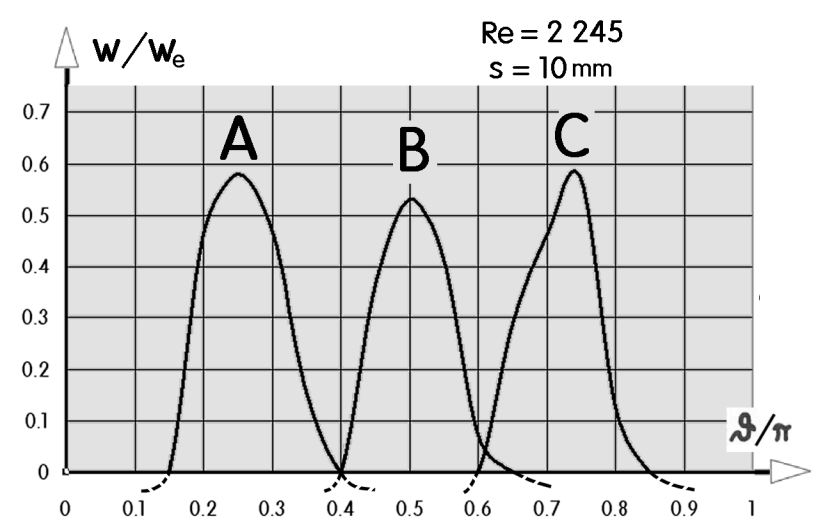

Figure 15. Pitot probe measurements of radial velocity $w$ in the three alternative stable regimes. Negative radial velocity (directed towards the disk, as obtained by computations in Figure 14) could not be measured by the Pitot probe. Velocity values are here non-dimensionalised by relating them to the nozzle exit velocity $\mathrm{w}_{\mathrm{e}}$.

observed in the symmetric flow configuration. The deflection was not limited to one side from the symmetry axis. A similar stationary deflection to the opposite side was also observed equally often. The discussed flowfield obviously exhibits three different stable flow regimes, labelled here $A, B$, and $C$. The distributions of the measured pressure on the disk surface in the three alternative regimes are presented in Fig. 14. The next Fig. 15 shows the measured profiles of radial velocity in the midplane between the PMMA endplates. Obviously, although not perfectly the same in Fig. 15 (deviations no doubt caused by manufacturing tolerances), all three generated jet configuration possess comparable total momentum flow rates (i.e. the jets in $A$ and $C$ configurations are not weaker than the symmetric jet flow B). Two facts of high importance are as follows: a) the flows $A$ and $C$ with the symmetry breaking were found only for the more pronounced curvature of the attached wall-jet flows - i.e. at the smaller relative curvature radius $r / b=10$, and $\mathbf{b})$ they are present only at low Reynolds numbers, less than $\operatorname{Re} \sim 3000$.
An also surprising fact - considering the success of the numerical flowfield computations in the symmetric case - was the impossibility of obtaining the asymmetric flowfields by computations. This may be the more surprising that symmetry was neither imposed in the mathematical model nor imposed by boundary conditions (as demonstrated in Fig. 8) and. the numerical solutions

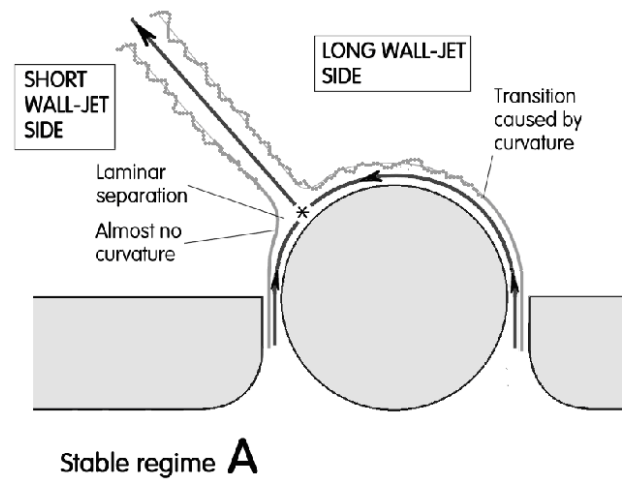

Figure 17. Schematic representation of the first author's hypothesis explaining the existence of the asymmetric regime $A$ (for which is valid this diagram) and its mirror image C.

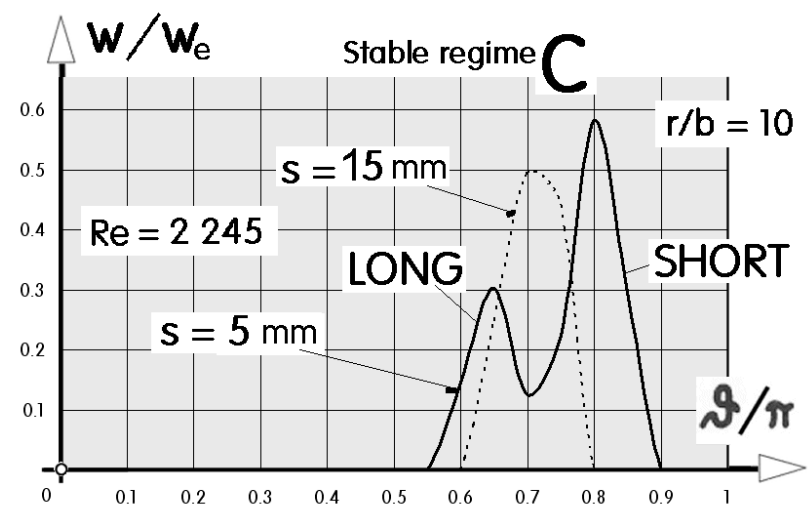

Figure 18. At small distances $s$ of the probe tip from the cylindrical attachment surface the velocity profiles have two peaks, each belonging to one of the two colliding jets. If the distance $\mathrm{s}$ is increased to $15 \mathrm{~mm}$ the two flows merge into a practically single jet (though of unusual character, demonstrated e.g. by the local minimum of fluctuation energy on jet axis, as shown in Figure 13).

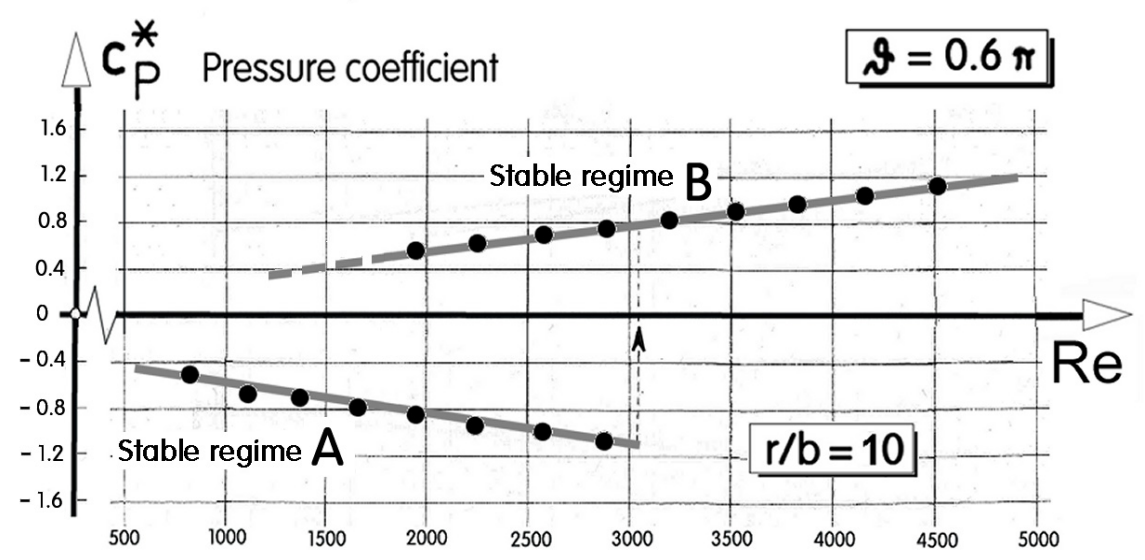

Figure 16. Linear dependences of the attachment-wall pressure coefficient $C_{P}^{*}$ - at a constant angular location $\boldsymbol{\$}=0.6 \pi-$ on varied Reynolds number. The existence of the regime $A$ only at low Reynolds numbers (less than $\operatorname{Re}=3000$ ) led to a jump change in the sign of the local pressure in response to gradual increase of the flow rate. 
were indeed able to show small asymmetries of the flowpaths as deviations from the symmetric flow. This fact suggests that the discovered phenomenon is associated with the problems and inadequateness of modelling the laminar/turbulent transition.

Since it is an already proved fact that the flow curvature causes earlier transition into turbulence, the existence of the symmetry breakdown effect only with the smaller one, $r / b=10$, of relative curvature radii is another support for the hypothesis that the phenomenon is a consequence of the transition, especially the curvatureenhanced one.

Finally, another observed fact that also supports the hypothesis is the experimental finding that the asymmetric flows exist only at low Reynolds numbers, below $\operatorname{Re} \sim 3$ 000. An example demonstrating this fact is presented in Fig. 16. It shows the measured values of the pressure coefficient $\mathrm{C}_{\mathrm{P}}{ }^{*}$ evaluated for different Reynolds numbers at a single angular location $\boldsymbol{N}=0.6 \pi$ in two flow regimes $A$ and $B$. The data may be compared

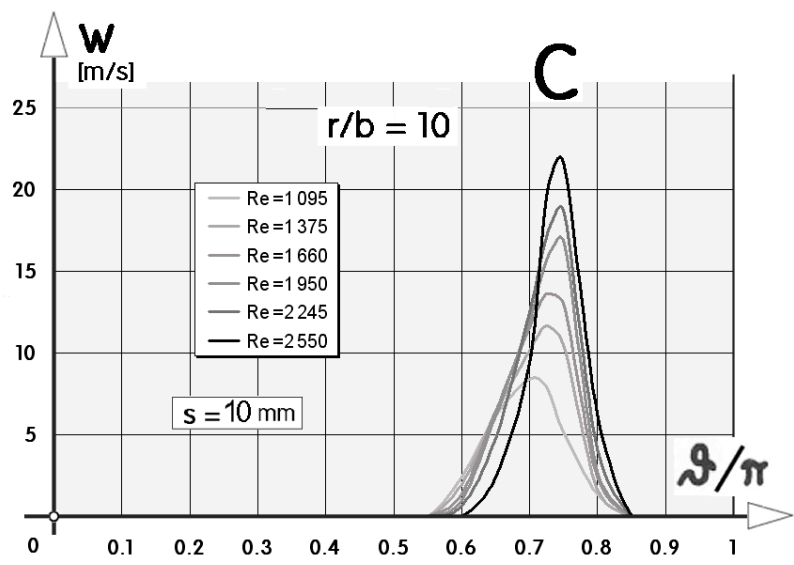

Figure 19. The character of the jet in the asymmetric flow regimes is here revealed by the measured profiles of radial velocity $\mathrm{W}$ at different Reynolds number conditions.

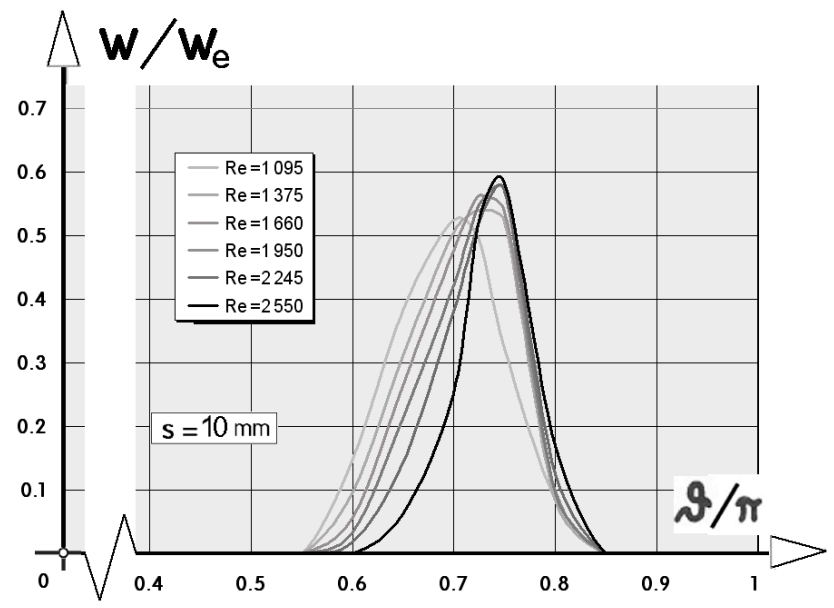

Figure 20. The velocity distributions in Figure 19 re-plotted into the relative vertical co-ordinate show the relatively small Reynolds number effect. with those in the diagram Figs. 7 and 14. The vertical line placed in Fig. 14 at $\boldsymbol{S}=0.6 \pi$ intersects the two curves for $A$ and $B$ so that in the case $A$ the coefficient $C_{P} *$ is negative (near to $C_{P} *=-1$ at the Reynolds number $R e=$ 2 865) while in the case $B$ the coefficient $C_{P} *$ is positive (near to $\left.C_{P} *=0.8\right)$. The corresponding positive and negative $C_{P} *$ values for the $A$ and $B$ regimes are seen in Fig. 16. The responses to varying Reynolds numbers were hysteretic, with jumping from $A$ to $B$ (shown by the dashed vertical line with the arrow).

Some characteristic features of the generated asymmetric air jet may be observed in the illustrations from Fig. 18 to 20, based on the performed measurements of velocity profiles.

\section{2 Explanation}

The colliding curved wall-jet flows in the asymmetric regime $A$ are presented schematically in Fig. 17. Let us assume that this deflected-jet asymmetric flow was obtained by some action on the resultant jet, forcing it to deflect. On the left-hand side of Fig. 17 the path from the nozzle to the stagnation point of the collision is short - its angular length according to Fig. 15 is about $0.25 \pi$. On the other, right-hand side the wall-jet flow is long, its path from the nozzle to the collision stagnation point is roughly $0.75 \pi$, i.e. three times as long. At the low Reynolds numbers of interest, both wall-jets are initially laminar. The conditions on the right-hand side are qualitatively similar to those in the symmetric flow: exposed to the influence of the curvature. The wall jet experiences an earlier transition into turbulence roughly at the indicated location. Turbulent wall-jet continuing from this location entrains outer air from atmosphere and this makes it (by the generated pressure difference) resistant to separation from the attachment wall. On the left-hand side, however, there is almost no curvature at the outer layers of the short wall jet. Without the enhanced transition, the wall-jet there remains laminar, the pressure difference is small — and this makes it disposed toward laminar separation.

It should be noted in Fig. 17 that the turbulent flow on the long jet side, while more resistant to the separation from the attachment wall, arrives in the collision stagnation point with lower momentum flow rate (having lost a considerable percentage by the turbulent momentum transport to the outer fluid and also, not so much, by wall friction). The laminar jet on the other, short side, is less resistant to the separation, but arrives in the stagnation point with higher momentum. The deflected position is rather stable due to the overall result of these positive and negative factors. As seen in Figs. 19 and 20, increasing Reynolds number causes the deflectedjet stagnation point position to move nearer to the nozzle - but the overall effect is not strong. 


\section{3 Tristable amplifier}

The most effective method how to put the stagnation point of the collision into the requested position $(A, B$, or $C)$ in the experiments was rotating the central disk with its pressure-tap slit to the corresponding angular position and then to blow into it a short air flow pulse. Instead of the rotation of the slit, it is possible to provide the disk (which then remains stationary, nontotated) with three suitably located slits a, b, and c, each with its input inlet chamber for the control flow pulse, as is shown in Fig. 21. With suitable dimensioning of the control inlets and rather small diameter of the central disk the control pulse may be not only short but also quite weak - and yet suffices for efficient control of the flowfield.

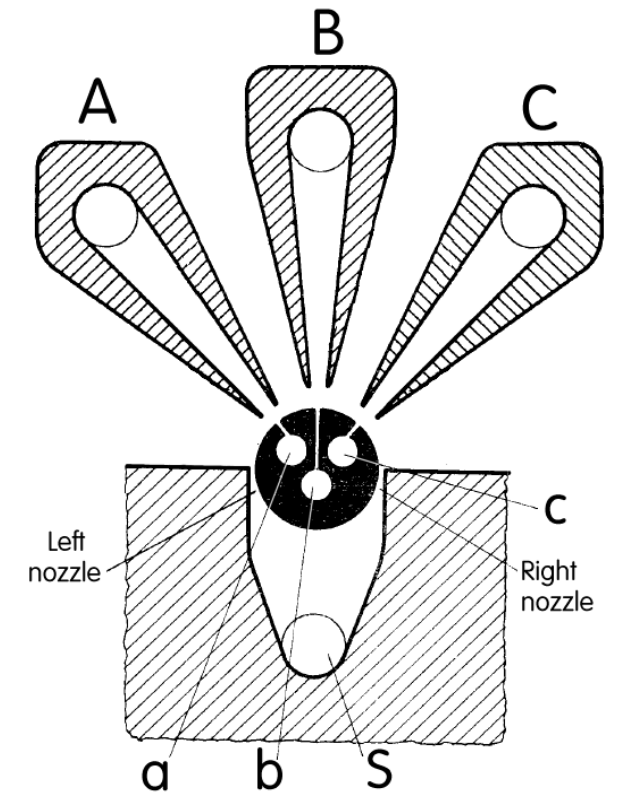

Figure 21. The three stable flow regimes $A, B$, and $C$ may be used in tri-stable fluidic devices - here in an amplifier [12] with switching the flow between the three regimes by a weak (and short) flow pulse admitted into one of the three control nozzle slits $\mathrm{a}, \mathrm{b}$, and $\mathrm{c}$.

Positioning three jet-capturing collectors in the corresponding locations shown in this picture, the device becomes tristable, remaining in any of the three stable positions until a change caused by another control pulse into another control slit. The idea of control systems operating with three regimes, for which this tristable fluidic amplifier is perfectly suited, is there for considerable time. The tree-valued logic is an extension of Boolean two-valued logic operating with three alternatively assumed values: true, false, and neither. One of the ancestors of digital computers, made as early as 1840 [9], operated in this ternary numeral system. A modern, electronic ternary computer was also built in 1958 by N. Brusentsov, claiming lower energy consumption and production cost lower than with common binary logic circuitry. The same advantages have led in 1973 to development of electronic ternary computer Ternac at State University of New York in Buffalo. Some computer theorists predict that elegance and efficiency of ternary logic will lead to its use in future computing [9].

\section{Conclusions}

Paper discusses results of experimental investigation and numerical flowfield computation of a flow - with two colliding curved wall-jets - apparently so far not yet studied. The high sensitivity to disturbances makes it a suitable candidate for being applied in fluidic sensors especially operating at quite low Reynolds numbers. Among the discovered features of the investigated flows perhaps the most interesting is the existence of symmetry breaking - an asymmetric flowfield generated in response to symmetric boundary conditions. This may be used in fluidic circuits working with three-valued logic.

\section{Acknowledgements}

This research was supported by research grant Nr. 1323046S obtained from GAČR. Authors also received institutional support RVO:61388998.

\section{References}

1. Z-G. Sun, et al., Industrial and Engineering Chemistry Research, 49, 5877 (2010)

2. S. Besbes, et al., Wärme- und Stoffübertragung, 39, 675 (2003)

3. P.M. Belotserkovskii, Izvestia Akad. Nauk, Mekhanika Zhidkosti i Gaza, 154, 1987

4. G.E. Myers, et al., Journal of Fluids Engineering, 85, 47 (1963)

5. R.J. Kind, K. Suthanthichan, Journal of Fluid Mechanics, 58, (1973)

6. P.S. Johansson, H. I. Anderson, Physics of Fluids, 17, 1 (2006)

7. V. Tesař, Flow Measurement and Instrumentation, 33, 2 (2013)

8. B.G. Newman, Canadian Aeronautics and Space Journal, 15, 287 (1969)

9. M. Glusker, et al., IEEE Annals of the History of Computing, 27, 4 (2005)

10. V. Tesař, Fluidic sensor of rotation speed, Czechoslovak Patent Nr. 148723 DOI: 10.13140/2.1.2548.6723, Oct. 1971

11. V. Tesař, Fluidic sensor of position or motion speed of mechanical components, Czechoslovak Patent Nr.166623 DOI: 10.13140/2.1.2548.6723, May 1975

12. V. Tesař, Tri-stable fluidic element, Czechoslovak Patent Nr. 162425, DOI: 10.13140/2.1.1085.8249, Feb. 1974

13. V. Tesař, Chemical Engineering Journal, 155, 789 (2009)

14. V. Tesař, Journal of Visualization, 6, 77 (2003)

15. V. Tesar̆, Flow Measurement and Instrumentation, 33, 228 (2013)

16. K. Peszynski, Diploma Thesis, Czech Technical Univ. in Prague, 1974 